\title{
The potential roles of circular RNAs in osteonecrosis of the femoral head (Review)
}

\author{
JUN ZHAO $^{1}$, LONG MU $^{2}$, ZHENGCHUN WANG $^{1}$, XIANGCHUN FANG $^{1}$, \\ XUEFENG $\mathrm{HE}^{1}$, XIAOFENG ZHANG $^{1}$ and XILIN XU ${ }^{1}$ \\ ${ }^{1}$ Department of Orthopaedics, Heilongjiang University of Chinese Medicine; \\ ${ }^{2}$ Department of Orthopaedics, Harbin Fifth Hospital, Harbin, Heilongjiang 150040, P.R. China
}

Received July 31, 2019; Accepted November 7, 2019

DOI: $10.3892 / \mathrm{mmr} .2019 .10866$

\begin{abstract}
Circular RNAs (circRNAs) are categorized as non-coding RNAs that, unlike widely known canonical linear RNAs, form a covalently closed continuous loop without $5^{\prime}$ or 3 ' polarities, which enables them to resist digestion by RNA exonucleases. Although the functions of circRNAs remain largely unknown, accumulated evidence has demonstrated that circRNAs can act as microRNA sponges, which allows them to regulate numerous biological processes and disease mechanisms, including apoptosis, angiogenesis, invasion, metastasis and stem cell differentiation. Although research into circRNAs is in its infancy, studies have identified critical roles for circRNAs in the initiation and progression of disease. The present study delineated the characteristics and functions of circRNAs, and focused on the potential relationship between circRNAs and osteonecrosis of the femoral head (ONFH). CircRNAs represent a novel avenue for studying the mechanisms underlying ONFH as well as possible treatments.
\end{abstract}

\section{Contents}

1. Introduction

2. Formation of circRNAs

3. Biological functions of circRNAs

4. CircRNAs and BMSCs

5. CircRNAs in osteoblasts and osteoclasts

6. CircRNAs and vascular endothelial cells

7. CircRNAs and chondrocytes

8. Conclusion and prospects

Correspondence to: Professor Xiaofeng Zhang, Department of Orthopaedics, Heilongjiang University of Chinese Medicine, 24 Heping Road, Xiangfang, Harbin, Heilongjiang 150040, P.R. China E-mail: 30793648@qq.com

Key words: circular RNA, osteonecrosis of the femoral head, bone marrow mesenchymal stem cells, osteoblasts and osteoclasts, vascular endothelial cells, chondrocytes

\section{Introduction}

In the 1970s, circRNAs, a unique closed circular form of non-coding RNA, were first discovered in plant viruses (1) and were considered a by-product of aberrant splicing reactions (2). In 2012, hundreds of circRNAs from different cell types were detected by RNA sequencing technology (3). With the development of high-throughput sequencing technology, several circRNAs have been detected in eukaryotes; however, they have been regarded as splicing by-products without biological functions (4). Unlike linear RNAs, which possess different 5 ' and $3^{\prime}$ ends that indicate the start and stop sites of transcription, circRNAs are the product of one or two exons being spliced together via covalent bonding of the $3^{\prime}$ and $5^{\prime}$ ends, resulting in establishment of a covalently closed continuous loop (4-7). Therefore, circRNAs are unlikely to be degraded by RNA exonuclease and are more stable than their linear counterparts (7-9); circRNA functions may be linked to their unique stability (9). Notably, previous studies have indicated that circRNAs can sponge microRNAs (miRNAs/miRs) or functional proteins to regulate specific biological functions at the transcriptional or post-transcriptional level (6,9-11). Therefore, circRNAs may be considered promising diagnostic biomarkers and therapeutic targets for numerous diseases.

Osteonecrosis of the femoral head (ONFH) is a disabling clinical disease that is most common among young adults (12). The mean age at diagnosis is typically $<50$ years (13). If no early intervention is provided, $\sim 80 \%$ of patients progress to femoral head collapse, hip dysfunction and permanent disability (14), and ultimately hip replacement becomes the only treatment option (13). However, postoperative infection, pain, functional rehabilitation, prosthetic replacement and other related complications (15-18) result in vast economic burdens for patients and for society. The mechanism by which ONFH develops remains unclear (19). The majority of experts agree that a lack of blood supply to the femoral head and bone marrow causes death of osteocytes, as explained by the microvascular injury theory, osteoporosis theory, apoptosis theory, osteogenic enhancement and adipogenic weakening theory of bone marrow mesenchymal stem cells (20-24). It has previously been reported that numerous circRNAs are associated with ONFH, which suggested that they may have a critical role in the development and progression of ONFH (25). This 
review aimed to describe the evidence regarding the biogenesis and biological functions of circRNAs, and to identify their potential mechanistic roles in ONFH.

\section{Formation of circRNAs}

The manner by which circRNAs are generated is different to how linear RNA transcripts are formed. Notably, the methods for generating circRNAs are either direct splicing or lasso-driven cyclization of precursor RNA, both with classical RNA polymerase-mediated splicing out of introns and connecting of exons via $5^{\prime}$ or $3^{\prime}$ polarities $(6,7,9,10)$. These two methods can form exon circRNAs (exon sequence only, ecircRNAs), intron circRNAs (composed of intron sequences, ciRNAs) and exon-intron circRNAs (exon and intron sequences, EIciRNAs) (Fig. 1); ecircRNAs account for $>80 \%$ of total circRNAs (26). The 3' end of the upstream exon of the precursor mRNA is covalently linked to the 5 ' end of the downstream exon by a reverse splicing process, after which the intron is cleaved to form an ecircRNA. If introns are retained, then EIciRNAs are formed (27). CiRNAs contain an exclusive 2'-5' linkage that distinguishes them from ecircRNAs, and their structure relies on $7 \mathrm{nt}$ GU-rich sequences near the 5 'splice site and C-rich sequences that are $11 \mathrm{nt}$ in length (28). Another novel model of circRNA biosynthesis has proposed that some RNA-binding proteins that have intron-binding abilities interact with intron-binding sites to promote the formation of circRNAs (29). Common RNA-binding proteins are protein quaking (29) and Drosophila muscle blind protein (30).

\section{Biological functions of circRNAs}

miRNA sponges. miRNAs are a class of non-coding RNA that contain $\sim 20$ nucleotides and bind to the 3'-untranslated region of mRNAs to inhibit their translation (31). Previous studies have offered evidence to suggest that some circRNAs act as aggressive endogenous RNAs that compete for miRNA-binding sites $(10,11,32)$. One study reported that circRNA E3 ubiquitin-protein ligase CHFR serves as a sponge for miR-370, which typically targets forkhead box protein (FOX)O1, and FOXO1 promotes the expression of cyclin D1 to drive the migration and proliferation of vascular smooth muscle cells. This provides an example of a profound role that circRNAs can serve in cardiovascular diseases (33). In addition, there are a number of overlapping binding sites between circRNAs and miRNAs, and a single circRNA can possibly interact with several miRNAs. For example, the mouse sex determination region $\mathrm{Y}$ is a testis-specific circRNA that has 16 binding sites and functions as a sponge for miR-138 (11), and circRNA homeodomain-interacting protein kinase 3 (circHIPK3) acts as a sponge for nine miRNAs (miR-654, miR-584, miR-379, etc.) and has 18 potential binding sites (34). However, due to the numerous binding possibilities between circRNAs and miRNAs, and the possibility of circRNAs interacting with multiple miRNAs, the specific mechanisms underlying the interactions between circRNAs and miRNAs requires further research.

Protein translation. As they possess a partial translation initiation codon and an open reading frame, the coding function of circRNAs has been confirmed by various studies $(35,36)$. Therefore, a comprehensive database of annotated human circRNAs has been constructed, which includes $\sim 33,000$ ecircRNAs (4). However, if an internal ribosome entry point is inserted upstream of the start codon of circRNAs, some proteins can be produced that are different from their linear transcripts (37). Legnini et al concluded that circRNA zinc finger protein 609 is associated with the process of muscle development and acts as a linear coding RNA that is translated to produce proteins, which provides a unique example of protein-coding circRNAs in eukaryotes (38). The translation of circRNAs has been confirmed and is closely related to the prognosis of certain diseases (39). Furthermore, it has been verified that circRNAs are modified by N6-methyladenosine (m6A), which induces base modification in mRNA, and there is one m6A site in circRNAs that is beneficial for promoting their translation (40).

Protein-binding functions. In addition to miRNAs, circRNAs also bind to proteins to modulate their corresponding functions. ciRNAs and EIciRNAs are mainly located in the nucleus and have unique tertiary structures that act as binding sites for RNA-binding proteins; therefore, these circRNAs can regulate gene transcription by directly interacting with RNA-binding proteins $(28,41)$. For example, circRNA FOXO3 (circFOXO3) inhibited cell cycle progression by binding to cyclin-dependent kinase 2 and cyclin-dependent kinase inhibitor 1, leading to the formation of a ternary complex (42). Notably, Du et al (43) demonstrated that overexpression of circFOXO3 reduced the binding between $\mathrm{FOXO} 3$ and murine double minute 2 (MDM2), and blunted the effect of MDM2 on modulating the polyubiquitination of FOXO3, which strengthened FOXO3 activity and promoted cell apoptosis (43). These findings suggested that the same circRNA may bind to different proteins in different tissues and cells to perform various functions.

It has been reported that circRNAs regulate alternative splicing, transcription, exosomal function and the formation of circRNA pseudogenes, all of which affect the occurrence and progression of disease $(6,30,44,45)$. Accumulating studies have demonstrated that circRNAs are highly associated with various diseases, such as cancer, kidney disease, diabetes, cardiovascular disease, Alzheimer's disease and osteoarthritis (OA) $(6,7,46)$. However, the relationship between circRNAs and the initiation of ONFH is still largely unclear; the present review provides a summary of what is currently known.

\section{CircRNAs and BMSCs}

Weakened osteogenic differentiation and increased adipogenic differentiation of BMSCs are closely associated with the formation of ONFH (47). miRNAs, which can be negatively regulated by the competitive binding of circRNAs, serve an extremely important role in regulating stem cell differentiation (48). Differential expression of 23 miRNAs has been identified in the BMSCs of steroid-induced ONFH (SONFH) (49). Furthermore, various studies have demonstrated that promoting osteogenic differentiation and inhibiting adipogenic differentiation of BMSCs by regulating the expression level of miRNAs can achieve the goal of effectively treating ONFH $(24,50,51)$. 


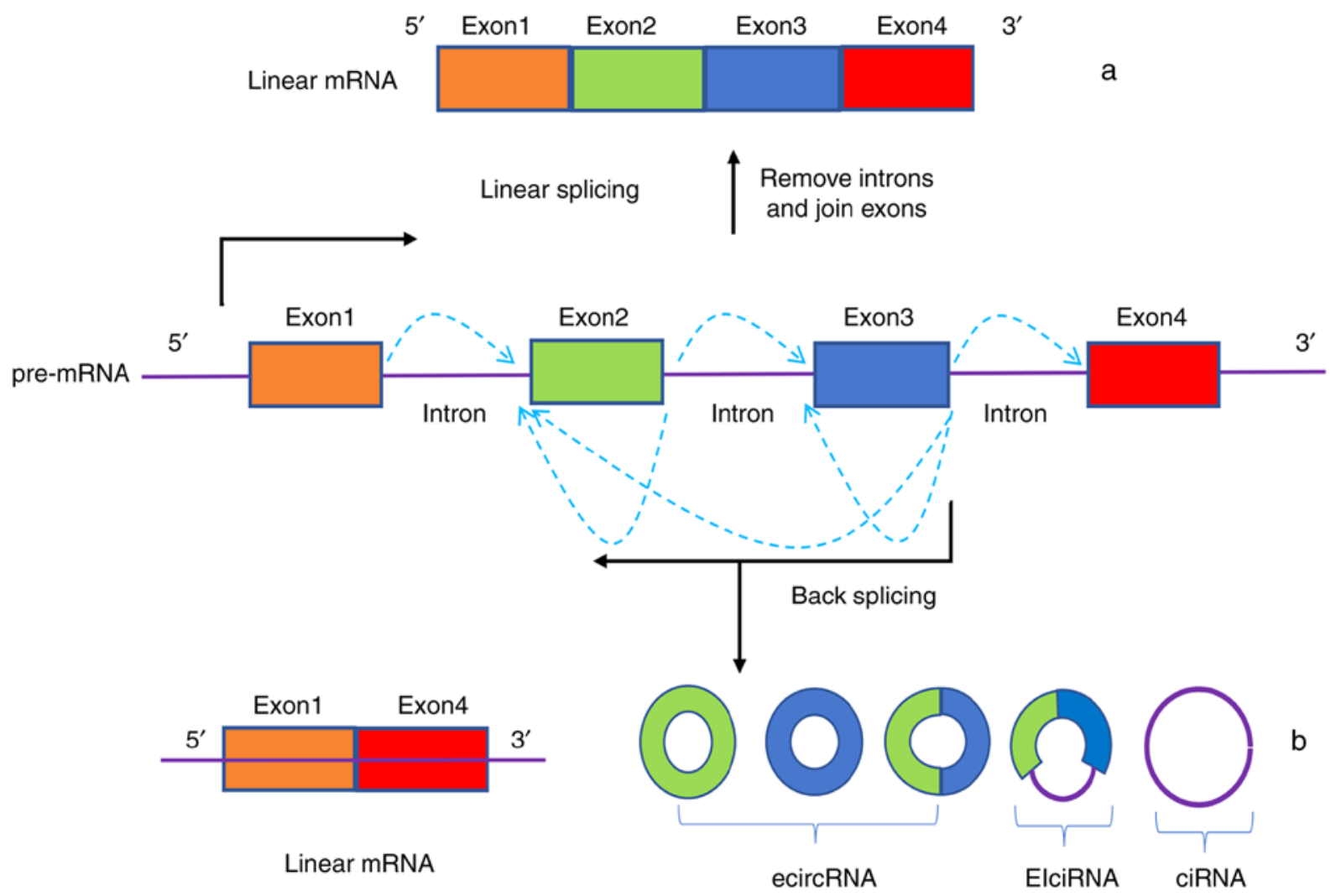

Figure 1. Different mechanisms for circRNA formation. (a) A constitutive linear RNA splicing pattern. (b) Formation of circRNAs can occur by direct splicing or by lasso-driven cyclization of precursor RNA. These two methods can produce ecircRNAs (exon sequence only), ciRNAs (composed of intron sequences) and EIciRNAs. ciRNA, intron circRNA; circRNA, circular RNA; ecircRNA, exon circRNA; EIciRNA, exon-intron circRNA.

A total of 231 preferentially expressed circRNAs were detected in the BMSCs of SONFH compared with the control group; 90 were upregulated and 141 were downregulated (25). CircRNAs were also differentially expressed during the osteogenic differentiation of BMSCs, $95 \%$ of which were protein-coding genes (52). In addition, silencing circRNA immunoglobulin superfamily member 11 promotes osteoblast differentiation and increases the expression of miR-199b-5p (52), which exerts its role in BMSC osteogenesis through the glycogen synthase kinase $3 \beta / \beta$-catenin signaling pathway (53) and acts as an important therapeutic targets during early-stage ONFH (54). A recent report indicated that circRNA FOXP1 acts as a sponge for several miRNAs and serves a pivotal role in the regulation of MSC differentiation (55). Additionally, Kuang et al (56) demonstrated that circRNA ubiquitin-specific protease 45 can upregulate the expression of phosphatase and tensin homolog through sponging miR-127-5p, which inhibits the protein kinase B pathway and regulates bone mass in rat SONFH. Therefore, circRNAs acting as miRNA sponges may be associated with osteogenic differentiation of BMSCs and ONFH. Although few studies have been performed on adipogenic differentiation, the aforementioned observations provide a good direction for studying circRNA, BMSCs and ONFH.

\section{CircRNAs in osteoblasts and osteoclasts}

Osteoblasts are the primary cells that function in bone formation, which are essential for mineralization of bone matrix (57). Osteoclasts secrete proteinases and hydrogen ions to degrade the organic bone matrix and dissolve bone minerals, respectively (57). Maintaining a balance between osteoblasts and osteoclasts is crucial for maintaining normal bone mass (58). Bone cell metabolism is irreversibly destroyed by local circulatory disorders, and leads to the disappearance of osteoblasts, activation of osteoclasts, the eventual destruction of trabecular bone and increased bone fragility (59). This destructive pathway was verified by the observation that osteoclast-related activity is increased in the subchondral bone and necrotic areas of ONFH tissues, whereas osteoblast activity is increased in the sclerotic region (60).

circRNA 19142 (circ19142) and circRNA 5846 (circ5846) act as sponges for miR-7067-5p; this is a network that is confirmed to function in osteoblast differentiation through a circ19142/circ5846-targeted miRNA-mRNA axis (61). Dou et al (62) reported that 19 circRNAs were upregulated and five circRNAs were downregulated during different stages of mouse osteoclast formation. In addition, 260 differentially expressed circRNAs were detected between peripheral blood lymphocytes from postmenopausal patients with osteoporosis and controls (63). It has also been demonstrated that the expression of circRNA 0001275 is markedly increased in postmenopausal patients with osteoporosis; therefore, it was concluded that it may be a potential diagnostic biomarker (64). In summary, it was hypothesized that circRNAs may have an important role in ONFH, which is closely related to the activity of osteoblasts and osteoclasts. Therefore, circRNAs may have a role as diagnostic biomarkers. Identifying circRNAs that 


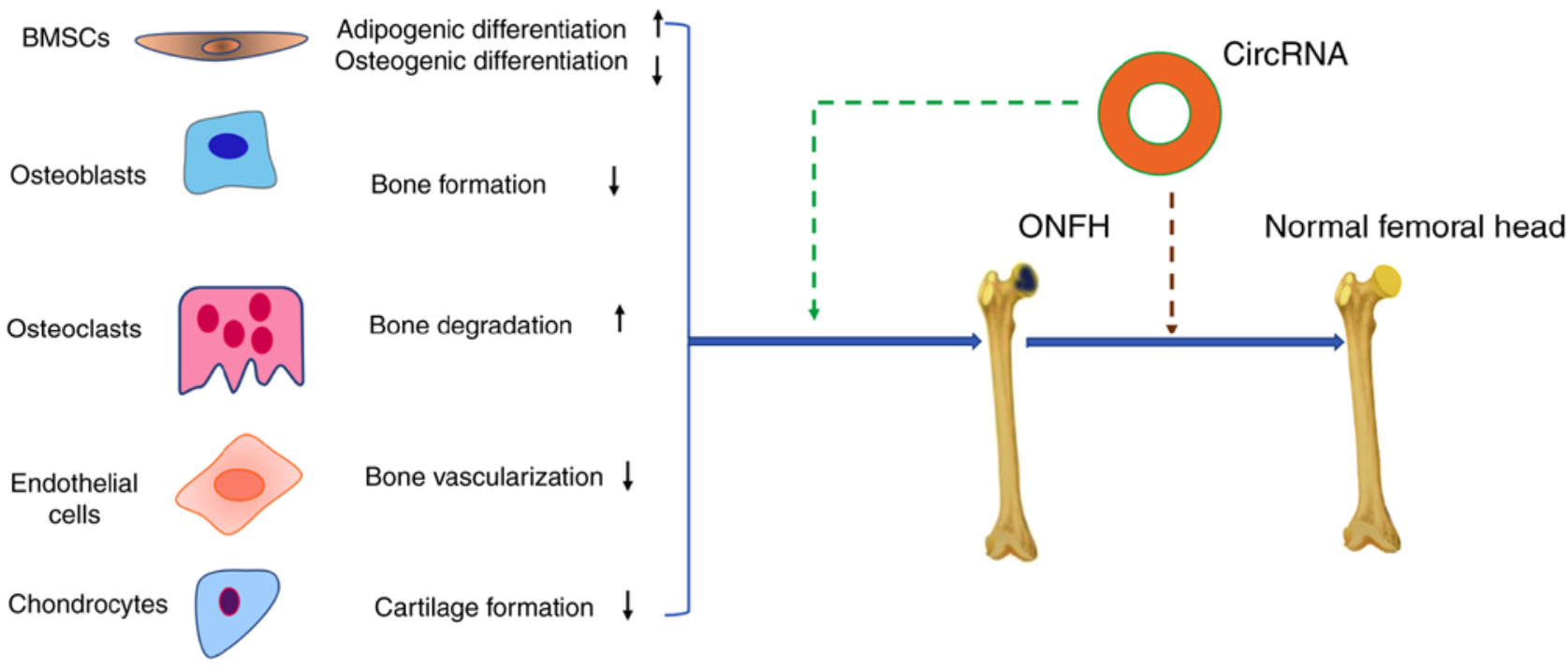

Figure 2. A variety of pathological mechanisms are involved in the formation of ONFH. circRNA-targeted treatment may be considered a novel method of ONFH therapy in the future. Black arrow: Up, indicates enhancement; down, indicates weakening. Dashed arrow: Green, indicates association and participation; red, indicates circRNA-targeted treatment. BMSCs, bone marrow mesenchymal stem cells; circRNA, circular RNA; ONFH, osteonecrosis of the femoral head.

are preferentially expressed in peripheral blood lymphocytes, during necrotic collapse or in sclerotic bone tissue may provide a novel direction for studying the relationship between circRNAs and ONFH.

\section{CircRNAs and vascular endothelial cells}

Endothelial cell damage, intravascular coagulation and disordered angiogenesis lead to ischemia, which is considered to be one of the core pathological results of ONFH $(20,22)$. Endothelial cell tube formation is the first step in neovascularization (65), and blood vessels are critical in bone remodeling because they supply nutrients (66). It has been reported that SONFH may cause miRNA alterations in femoral head bone microvascular endothelial cells that are not seen in controls (67). The vascularity of the bone in osteonecrosis is reduced by $\sim 50 \%$ (68). Additionally, a reduction in bone strength and vascularity of the bone precedes bone mass reduction and microstructural deterioration (69). Previous studies have suggested that ameliorating vascular endothelial cell proliferation, migration and tube formation may positively promote bone vascularization in the femoral head and prevent ONFH (70,71).

Notably, circRNAs are expressed in endothelial cells and serve a biological role in angiogenesis (72). For example, circRNA 0003575 is upregulated in oxidized low-density lipoprotein-induced human umbilical vein endothelial cells (HUVECs), and promotes HUVEC proliferation and angiogenesis (73). CircRNA 0010729 mediates vascular endothelial cell apoptosis and proliferation by targeting the miR-186/hypoxia inducible factor-1 $\alpha$ axis (74). Furthermore, Shan et al (75) reported that circHIPK3 expression is substantially upregulated in endothelial cells during diabetic retinal vasculopathy, and in vitro endothelial cell viability, proliferation, migration, and tube formation are altered by silencing or overexpressing circHIPK3. Acting as an endogenous miR-30a-3p sponge, in vivo silencing of circHIPK3 also attenuates retinal vascular dysfunction (75). Therefore, it was hypothesized that circRNAs may be involved in the endothelial cell damage and disruption of angiogenesis that lead to the formation of ONFH; circRNAs may be considered potential therapeutic targets.

\section{CircRNAs and chondrocytes}

It is generally believed that structural damage of the subchondral bone in post-collapse cases of osteonecrosis contributes to degeneration of articular cartilage (76). Notably, at the beginning of ONFH, the subchondral bone receives reduced nutrition because the blood supply to the area is impaired; that, coupled with degeneration of the cartilage matrix, leads to degeneration of articular cartilage, which can increase instability of the hip joint and accelerate the development of ONFH $(77,78)$. Therefore, prevention and early treatment of hip cartilage damage are beneficial for ameliorating the progression of ONFH (78).

Cartilage inflammation can be enhanced by interleukin-21, which causes degradation of the cartilage in patients with ONFH through the Janus kinase/signal transducers and activators of transcription signaling pathway (79). However, studies on the role of circRNAs in cartilage degeneration are currently more focused on OA $(80,81)$. In addition, it has been reported that intermittent cyclic mechanical tension leads to differential expression of miRNAs, which regulates stromal metabolism and calcification of cartilage endplate chondrocytes via the transforming growth factor- $\beta$ signaling pathway (82). Furthermore, differentially expressed circRNAs are detected in different regions of cartilage in patients with OA; circRNA 100226 is associated with mechanical tension, and it was demonstrated that circRNA 100226, acting as a sponge for miR-875, promotes the degradation of cartilage matrix by controlling the expression of tumor necrosis factor $\alpha$ (83). Therefore, it was concluded that mechanical stress changes in the early stages and in the collapsed areas of ONFH may lead to alterations in the expression of miRNAs 
and circRNAs in the corresponding regional cartilage. The molecular mechanism underlying the function of circRNAs in the degradation of ONFH cartilage damage is essential for understanding the pathogenesis of ONFH and exploring novel prospective therapeutic targets.

\section{Conclusion and prospects}

CircRNAs were discovered decades ago. With advances in research methods, increasing attention has been paid to circRNAs. Notably, it has been reported that circRNAs are involved in the development and progression of various diseases $(6,7,46)$. Although differential expression of circRNAs was observed in BMSCs of ONFH and a possible connection was suggested (25), to the best of our knowledge, no further functional or molecular mechanisms have been investigated. With respect to the potential pathological mechanisms of $\mathrm{ONFH}$, this review describes the potential relationship between BMSCs, osteoblasts, osteoclasts, vascular endothelial cells, articular cartilage and circRNAs (Fig. 2). Addressing these problems may be beneficial to the prevention of ONFH and the development of therapeutic targets for its treatment.

CircRNAs can function as miRNA sponges, regulate gene transcription and interact with RNA-binding proteins $(10,11,28,29)$. Most circRNA studies focus on their role as miRNA sponges, that is, the related mechanisms and functions of the circRNA-miRNA-mRNA axis in human diseases (84). However, a single circRNA has numerous binding sites for one miRNA and can interact with multiple miRNAs; therefore, more extensive research is required to illuminate the functional interactions between circRNAs and miRNAs. In addition, the role of circRNAs in regulating protein translation and binding to RNA-binding proteins in various diseases requires additional research.

In conclusion, circRNAs have a potential role in the initiation mechanisms of ONFH, and they exhibit high stability compared with linear RNAs. Therefore, it may be hypothesized that circRNAs serve a unique role in the formation $\mathrm{ONFH}$ and could have a role in ONFH therapy.

\section{Acknowledgements}

Not applicable.

\section{Funding}

No funding was received.

\section{Availability of data and materials}

Not applicable.

\section{Authors' contributions}

JZ was responsible for reviewing the concept design, and wrote and proofread the article. LM created the figures and made important comments on the revision of the article. $\mathrm{ZW}, \mathrm{XF}$, $\mathrm{XH}, \mathrm{XZ}$ and XX participated in literature collection, analysis and summary. XZ was responsible for project guidance. All authors read and agree to the final text.

\section{Ethics approval and consent to participate}

Not applicable.

\section{Patient consent for publication}

Not applicable.

\section{Competing interests}

The authors declare that they have no competing interests.

\section{References}

1. Sanger HL, Klotz G, Riesner D, Gross HJ and Kleinschmidt AK: Viroids are single-stranded covalently closed circular RNA molecules existing as highly base-paired rod-like structures. Proc Natl Acad Sci USA 73: 3852-3856, 1976.

2. Cocquerelle C, Mascrez B, Hétuin D and Bailleul B: Mis-splicing yields circular RNA molecules. FASEB J 7: 155-160, 1993.

3. Salzman J, Gawad C, Wang PL, Lacayo N and Brown PO: Circular RNAs are the predominant transcript isoform from hundreds of human genes in diverse cell types. PLoS One 7: e30733, 2012.

4. Chen X, Han P, Zhou T, Guo X, Song X and Li Y: circRNADb: A comprehensive database for human circular RNAs with protein-coding annotations. Sci Rep 6: 34985, 2016.

5. You X, Vlatkovic I, Babic A, Will T, Epstein I, Tushev G, Akbalik G, Wang M, Glock C, Quedenau C, et al: Neural circular RNAs are derived from synaptic genes and regulated by development and plasticity. Nat Neurosci 18: 603-610, 2015.

6. Greene J, Baird AM, Brady L, Lim M, Gray SG, McDermott R and Finn SP: Circular RNAs: Biogenesis, function and role in human diseases. Front Mol Biosci 4: 38, 2017.

7. Aufiero S, Reckman YJ, Pinto YM and Creemers EE: Circular RNAs open a new chapter in cardiovascular biology. Nat Rev Cardiol 16: 503-514, 2019.

8. Enuka Y, Lauriola M, Feldman ME, Sas-Chen A, Ulitsky I and Yarden Y: Circular RNAs are long-lived and display only minimal early alterations in response to a growth factor. Nucleic Acids Res 44: 1370-1383, 2016.

9. Müller S and Appel B: In vitro circularization of RNA. RNA Biol 14: 1018-1027, 2017.

10. Memczak S, Jens M, Elefsinioti A, Torti F, Krueger J, Rybak A, Maier L, Mackowiak SD, Gregersen LH, Munschauer M, et al: Circular RNAs are a large class of animal RNAs with regulatory potency. Nature 495: 333-338, 2013.

11. Hansen TB, Jensen TI, Clausen BH, Bramsen JB, Finsen B, Damgaard CK and Kjems J: Natural RNA circles function as efficient microRNA sponges. Nature 495: 384-388, 2013.

12. Cui L, Zhuang Q, Lin J, Jin J, Zhang K, Cao L, Lin J, Yan S, Guo W, He W, et al: Multicentric epidemiologic study on six thousand three hundred and ninety five cases of femoral head osteonecrosis in China. Int Orthop 40: 267-276, 2016.

13. Lespasio MJ, Sodhi N and Mont MA: Osteonecrosis of the Hip: A primer. Perm J 23: 18-100, 2019.

14. Kuroda Y, Matsuda S and Akiyama H: Joint-preserving regenerative therapy for patients with early-stage osteonecrosis of the femoral head. Inflamm Regen 36: 4, 2016.

15. Mufarrih SH, Qureshi NQ, Sadruddin A,Hashmi P, Mahmood SF, Zafar A and Noordin S: Relationship between staphylococcus aureus carriage and surgical site infections following total hip and knee arthroplasty in the South Asian Population: Protocol for a prospective cohort study. JMIR Res Protoc 7: e10219, 2018

16. Robertson-Waters E, Berstock JR, Whitehouse MR and Blom AW: Surgery for greater trochanteric pain syndrome after total hip replacement confers a poor outcome. Int Orthop 42: 77-85, 2018.

17. Bandholm T, Wainwright TW and Kehlet H: Rehabilitation strategies for optimisation of functional recovery after major joint replacement. J Exp Orthop 5: 44, 2018.

18. Hauer G, Vielgut I, Amerstorfer F, Maurer-Ertl W, Leithner A and Sadoghi P: Survival rate of Short-stem hip prostheses: A comparative analysis of clinical studies and national arthroplasty registers. J Arthroplasty 33: 1800-1805, 2018. 
19. Arbab D and König DP: Atraumatic femoral head necrosis in adults. Dtsch Arztebl Int 113: 31-38, 2016.

20. Zhang Q, L VJ and Jin L: Role of coagulopathy in glucocorticoid-induced osteonecrosis of the femoral head. J Int Med Res 46: 2141-2148, 2018.

21. Petek D, Hannouche D and Suva D: Osteonecrosis of the femoral head: Pathophysiology and current concepts of treatment. EFORT Open Rev 4: 85-97, 2019.

22. Kerachian MA, Séguin C and Harvey EJ: Glucocorticoids in osteonecrosis of the femoral head: A new understanding of the mechanisms of action. J Steroid Biochem Mol Biol 114: 121-128, 2009.

23. Zalavras C, Shah S, Birnbaum MJ and Frenkel B: Role of apoptosis in glucocorticoid-induced osteoporosis and osteonecrosis Crit Rev Eukaryot Gene Expr 13: 221-235, 2003.

24. Zhun W, Donghai L, Zhouyuan Y, Haiyan Z and Pengde K: Efficiency of cell therapy to GC-induced ONFH: BMSCs with Dkk-1 interference is not superior to unmodified BMSCs. Stem Cells Int 2018: 1340252, 2018

25. Xiang Shuai: The study of changed biological behavior and aberrantly expressed transcriptome in BMSCs in seroid-induced osteonecrosis (D). Peking Union Medical College, 2018.

26. Wang W, Wang Y, Piao H, Li B, Huang M, Zhu Z, Li D, Wang T, $\mathrm{Xu} \mathrm{R}$ and Liu K: Circular RNAs as potential biomarkers and therapeutics for cardiovascular disease. PeerJ 7: e6831, 2019.

27. Zhang XO, Dong R, Zhang Y, Zhang JL, Luo Z, Zhang J, Chen LL and Yang L: Diverse alternative back-splicing and alternative splicing landscape of circular RNAs. Genome Res 26 : $1277-1287,2016$

28. Li Z, Huang C, Bao C, Chen L, Lin M, Wang X, Zhong G, Yu B, Hu W, Dai L, et al: Exon-intron circular RNAs regulate transcription in the nucleus. Nat Struct Mol Biol 22: 256-264, 2015.

29. Conn SJ, Pillman KA, Toubia J, Conn VM, Salmanidis M, Phillips CA, Roslan S, Schreiber AW, Gregory PA and Goodall GJ: The RNA binding protein quaking regulates formation of circRNAs. Cell 160: 1125-1134, 2015.

30. Ashwal-Fluss R, Meyer M, Pamudurti NR, Ivanov A, Bartok O, Hanan M, Evantal N, Memczak S, Rajewsky N and Kadener S: circRNA biogenesis competes with pre-mRNA splicing. Mol Cell 56: 55-66, 2014.

31. Bartel DP: MicroRNAs: Target recognition and regulatory functions. Cell 136: 215-233, 2009

32. Kulcheski FR, Christoff AP and Margis R: Circular RNAs are miRNA sponges and can be used as a new class of biomarker. J Biotechnol 238: 42-51, 2016.

33. Yang L, Yang F, Zhao H, Wang M and Zhang Y: Circular RNA circCHFR facilitates the proliferation and migration of vascular smooth muscle via miR-370/FOXO1/Cyclin D1 pathway. Mol Ther Nucleic Acids 16: 434-441, 2019.

34. Zheng Q, Bao C, Guo W, Li S, Chen J, Chen B, Luo Y, Lyu D, $\mathrm{Li} Y$, Shi G, et al: Circular RNA profiling reveals an abundant circHIPK3 that regulates cell growth by sponging multiple miRNAs. Nat Commun 7: 11215, 2016.

35. Pamudurti NR, Bartok O, Jens M, Ashwal-Fluss R, Stottmeister C, Ruhe L, Hanan M, Wyler E, Perez-Hernandez D, Ramberger E, et al: Translation of CircRNAs. Mol Cell 66: 9-21. e27, 2017.

36. Abe N, Matsumoto K, Nishihara M, Nakano Y, Shibata A, Maruyama H, Shuto S, Matsuda A, Yoshida M, Ito Y and Abe H: Rolling circle translation of circular RNA in living human cells. Sci Rep 5: 16435, 2015

37. Wu J, Qi X, Liu L, Hu X, Liu J, Yang J, Yang J, Lu L, Zhang Z, $\mathrm{Ma} S$, et al: Emerging epigenetic regulation of circular RNAs in human cancer. Mol Ther Nucleic Acids 16: 589-596, 2019.

38. Legnini I, Di Timoteo G, Rossi F, Morlando M, Briganti F, Sthandier O, Fatica A, Santini T, Andronache A, Wade M, et al: Circ-ZNF609 is a circular RNA that can be translated and functions in myogenesis. Mol Cell 66: 22-37.e9, 2017.

39. Yang Y, Gao X, Zhang M, Yan S, Sun C, Xiao F, Huang N, Yang X, Zhao K, Zhou H, et al: Novel role of FBXW7 circular RNA in repressing Glioma tumorigenesis. J Natl Cancer Inst: 110, 2018 doi: 10.1093/jnci/djx166

40. Yang Y, Fan X, Mao M, Song X, Wu P, Zhang Y, Jin Y, Yang Y, Chen LL, Wang Y, et al: Extensive translation of circular RNAs driven by $\mathrm{N}^{6}$-methyladenosine. Cell Res 27 626-641, 2017.

41. Du WW, Zhang C, Yang W, Yong T, Awan FM and Yang BB: Identifying and Characterizing circRNA-protein interaction. Theranostics 7: 4183-4191, 2017.
42. Du WW, Yang W, Liu E, Yang Z, Dhaliwal P and Yang BB Foxo3 circular RNA retards cell cycle progression via forming ternary complexes with p21 and CDK2. Nucleic Acids Res 44: 2846-2858, 2016

43. Du WW, Fang L, Yang W, Wu N, Awan FM, Yang Z and Yang BB: Induction of tumor apoptosis through a circular RNA enhancing Foxo3 activity. Cell Death Differ 24: 357-370, 2017.

44. Li Y, Zheng Q, Bao C, Li S, Guo W, Zhao J, Chen D, Gu J, He X and Huang S: Circular RNA is enriched and stable in exosomes: A promising biomarker for cancer diagnosis. Cell Res 25 981-984, 2015

45. Dong R, Zhang XO, Zhang Y, Ma XK, Chen LL and Yang L: CircRNA-derived pseudogenes. Cell Res 26: 747-750, 2016.

46. Yu CX and Sun S: An emerging role for circular RNAs in osteoarthritis. Yonsei Med J 59: 349-355, 2018.

47. Houdek MT, Wyles CC, Packard BD, Terzic A, Behfar A and Sierra RJ: Decreased osteogenic activity of mesenchymal stem cells in patients with corticosteroid-induced osteonecrosis of the femoral head. J Arthroplasty 31: 893-898, 2016.

48. Ong SG, Lee WH, Kodo K and Wu JC: MicroRNA-mediated regulation of differentiation and trans-differentiation in stem cells. Adv Drug Deliv Rev 88: 3-15, 2015.

49. Wang B, Yu P, Li T, Bian Y and Weng X: MicroRNA expression in bone marrow mesenchymal stem cells from mice with steroid-induced osteonecrosis of the femoral head. Mol Med Rep 12: 7447-7454, 2015.

50. Li R, Lin QX, Liang XZ, Liu GB, Tang H, Wang Y, Lu SB and Peng J: Stem cell therapy for treating osteonecrosis of the femoral head: From clinical applications to related basic research. Stem Cell Res Ther 9: 291, 2018.

51. Gu C, Xu Y,Zhang S, Guan H, Song S, Wang X, Wang Y, Li Y and Zhao G: miR-27a attenuates adipogenesis and promotes osteogenesis in steroid-induced rat BMSCs by targeting PPARgamma and GREM1. Sci Rep 6: 38491, 2016.

52. Zhang M, Jia L and Zheng Y: circRNA expression profiles in human bone marrow stem cells undergoing osteoblast differentiation. Stem Cell Rev 15: 126-138, 2019.

53. Zhao R, Li Y, Lin Z, Wan J, Xu C, Zeng Y and Zhu Y: miR-199b-5p modulates BMSC osteogenesis via suppressing GSK-3 $\beta / \beta$-catenin signaling pathway. Biochem Biophys Res Commun 477: 749-754, 2016.

54. Huang L, Wang Y, Jiang Y, Wu Y, Hu C and Ouyang H: High levels of GSK-3 $\beta$ signalling reduce osteogenic differentiation of stem cells in osteonecrosis of femoral head. J Biochem 163: 243-251, 2018

55. Cherubini A, Barilani M, Rossi RL, Jalal MMK, Rusconi F, Buono G, Ragni E, Cantarella G, Simpson H, Péault B and Lazzari L: FOXP1 circular RNA sustains mesenchymal stem cell identity via microRNA inhibition. Nucleic Acids Res 47: 5325-5340, 2019.

56. Kuang MJ, Xing F, Wang D, Sun L, Ma JX and Ma XL: CircUSP45 inhibited osteogenesis in glucocorticoid-induced osteonecrosis of femoral head by sponging miR-127-5p through PTEN/AKT signal pathway: Experimental studies. Biochem Biophys Res Commun 509: 255-261, 2019.

57. Mandelin J, Hukkanen M, Li TF, Korhonen M, LiljeströmM, Sillat T, Hanaemaijer R, Salo J, Santavirta S and Konttinen YT: Human osteoblasts produce cathepsin K. Bone 38: 769-777, 2006.

58. Chen X, Wang Z, Duan N, Zhu G, Schwarz EM and Xie C: Osteoblast-osteoclast interactions. Connect Tissue Res 59: 99-107, 2018

59. Wang C, Wang X, Xu XL, Yuan XL, Gou WL, Wang AY, Guo QY, Peng J and Lu SB: Bone microstructure and regional distribution of osteoblast and osteoclast activity in the osteonecrotic femoral head. PLoS One 9: e96361, 2014

60. Wang C, Meng H, Wang Y, Zhao B, Zhao C, Sun W, Zhu Y, Han B, Yuan X, Liu R, et al: Analysis of early stage osteonecrosis of the human femoral head and the mechanism of femoral head collapse. Int J Biol Sci 14: 156-164, 2018.

61. Qian DY, Yan GB, Bai B, Chen Y, Zhang SJ, Yao YC and Xia H: Differential circRNA expression profiles during the BMP2-induced osteogenic differentiation of MC3T3-E1 cells. Biomed Pharmacother 90: 492-499, 2017

62. Dou C, Cao Z, Yang B, Ding N, Hou T, Luo F, Kang F, Li J, Yang X, Jiang H, et al: Changing expression profiles of lncRNAs, mRNAs, circRNAs and miRNAs during osteoclastogenesis. Sci Rep 6: 21499, 2016.

63. Jin D, Wu X, Yu H, Jiang L, Zhou P, Yao X, Meng J, Wang L, Zhang $M$ and Zhang Y: Systematic analysis of IncRNAs, mRNAs, circRNAs and miRNAs in patients with postmenopausal osteoporosis. Am J Transl Res 10: 1498-1510, 2018. 
64. Zhao K, Zhao Q, Guo Z, Chen Z, Hu Y, Su J, Chen L, He Z, Cai X, Chen M, et al: Hsa_Circ_0001275: A potential novel diagnostic biomarker for postmenopausal osteoporosis. Cell Physiol Biochem 46: 2508-2516, 2018.

65. Carulli $\mathrm{C}$, Innocenti $\mathrm{M}$ and Brandi ML: Bone vascularization in normal and disease conditions. Front Endocrinol (Lausanne) 4: $106,2013$.

66. Sivaraj KK and Adams RH: Blood vessel formation and function in bone. Development 143: 2706-2715, 2016.

67. Yue J, Wan F, Zhang Q, Wen P, Cheng L, Li P and Guo W: Effect of glucocorticoids on miRNA expression spectrum of rat femoral head microcirculation endothelial cells. Gene 651: 126-133, 2018

68. Lane NE: Glucocorticoid-induced osteoporosis: New insights into the pathophysiology and treatments. Curr Osteoporos Rep 17: 1-7, 2019.

69. Weinstein RS, Hogan EA, Borrelli MJ, Liachenko S, O'Brien CA and Manolagas SC: The pathophysiological sequence of glucocorticoid-induced osteonecrosis of the femoral head in male mice. Endocrinology 158: 3817-3831, 2017.

70. Liu X, Li Q, Niu X, Hu B, Chen S, Song W, Ding J, Zhang C and Wang Y: Exosomes secreted from human-induced pluripotent stem cell-derived mesenchymal stem cells prevent osteonecrosis of the femoral head by promoting angiogenesis. Int J Biol Sci 13 : 232-244, 2017

71. Zhang Y, Yin J, Ding H, Zhang C and Gao YS: Vitamin K2 ameliorates damage of blood vessels by glucocorticoid: A potential mechanism for its protective effects in glucocorticoid-induced osteonecrosis of the femoral head in a rat model. Int J Biol Sci 12 . 776-785, 2016

72. Boeckel JN, Jaé N, Heumüller AW, Chen W, Boon RA, Stellos K, Zeiher AM, John D, Uchida S and Dimmeler S: Identification and characterization of hypoxia-regulated endothelial circular RNA. Circ Res 117: 884-890, 2015.

73. Li CY, Ma L and Yu B: Circular RNA hsa_circ_0003575 regulates oxLDL induced vascular endothelial cells proliferation and angiogenesis. Biomed Pharmacother 95: 1514-1519, 2017.

74. Dang RY, Liu FL and Li Y: Circular RNA hsa_circ_0010729 regulates vascular endothelial cell proliferation and apoptosis by targeting the miR-186/HIF-1 $\alpha$ axis. Biochem Biophys Res Commun 490: 104-110, 2017.

75. Shan K, Liu C, Liu BH, Chen X, Dong R, Liu X, Zhang YY, Liu B, Zhang SJ, Wang JJ, et al: Circular noncoding RNA HIPK3 mediates retinal vascular dysfunction in diabetes mellitus. Circulation 136: 1629-1642, 2017.
76. Magnussen RA, Guilak F and Vail TP: Cartilage degeneration in post-collapse cases of osteonecrosis of the human femoral head: Altered mechanical properties in tension, compression, and shear. J Orthop Res 23: 576-583, 2005.

77. Chen G, Zhong L, Wang Q, Li Z, Shang J, Yang Q, Du Z, Wang J, Song Y and Zhang G: The expression of chondrogenesis-related and arthritis-related genes in human ONFH cartilage with different Ficat stages. PeerJ 7: e6306, 2019.

78. Xu R, Wei B, Li J, Huang C, Lin R, Tang C, Xu Y, Yao Q and Wang L: Investigations of cartilage matrix degeneration in patients with Early-stage femoral head necrosis. Med Sci Monit 23: 5783-5792, 2017.

79. Chen B, Liu Y and Cheng L: IL-21 enhances the degradation of cartilage through the JAK-STAT signaling pathway during osteonecrosis of femoral head cartilage. Inflammation 41: 595-605, 2018.

80. Zhou Z, Du D, Chen A and Zhu L: Circular RNA expression profile of articular chondrocytes in an IL-1 $\beta$-induced mouse model of osteoarthritis. Gene 644: 20-26, 2018

81. Shen S, Wu Y, Chen J, Xie Z, Huang K, Wang G, Yang Y, Ni W, Chen Z, Shi P, et al: CircSERPINE2 protects against osteoarthritis by targeting miR-1271 and ETS-related gene. Ann Rheum Dis 78: 826-836, 2019.

82. Feng C, Liu M, Fan X, Yang M, Liu H and Zhou Y: Intermittent cyclic mechanical tension altered the microRNA expression profile of human cartilage endplate chondrocytes. Mol Med Rep 17: 5238-5246, 2018.

83. Liu Q, Zhang X, Hu X, Yuan L, Cheng J, Jiang Y and Ao Y: Emerging roles of circRNA related to the mechanical stress in human cartilage degradation of osteoarthritis. Mol Ther Nucleic Acids 7: 223-230, 2017

84. Rong D, Su H, Li Z, Liu S, Dong C, Fu K, Tang W and Cao H: An emerging function of circRNA-miRNAs-mRNA axis in human diseases. Oncotarget 8: 73271-73281, 2017.

This work is licensed under a Creative Commons Attribution-NonCommercial-NoDerivatives 4.0 International (CC BY-NC-ND 4.0) License. 